\title{
Disfunção Cognitiva na Depressão: O Triste Esquecimento
}

\section{Cognitive Dysfunction in Depression: The Sad Forgetfulness}

Raquel Lobo Cardosoㄹ, Leonor Luz Duarte², Ana Monteiro³, Vítor Tedim Cruz, Joana Vitória-Silva ${ }^{5}$, Filipa Ramalho e Silva ${ }^{5}$

Autor Correspondente/Corresponding Author: Raquel Alexandra Lobo Cardoso [raquellobocardoso@gmail.com] Rua de Alfredo Cunha 365, 4450-01 Matosinhos, Portugal ORCID-iD: 0000-0003-0841-2555

\section{RESUMO}

INTRODUÇÃO: Aproximadamente 30\% a 40\% dos idosos com depressão apresentam sintomas cognitivos reversíveis, vulgarmente denominada de pseudodemência, que corresponde à condição tratável mais vezes confundida com demência.

O nosso objetivo foi rever a evidência existente sobre a disfunção cognitiva na depressão e as principais diferenças no diagnóstico, abordagem e prognóstico, comparativamente à demência.

METOdologiA: Revisão da literatura usando os seguintes termos MeSH: "Depressive Disorder", "Cognitive Dysfunction" e "Dementia".

RESULTADOS E CONCLUSÃO: Diversas situações podem cursar com défices cognitivos, sendo essencial colher uma história clínica completa e avaliar o estado funcional basal do doente. O exame neurológico deve ser sempre realizado, assim como excluídas as principais causas orgânicas de défice cognitivo. O papel do médico de família é importante na orientação do doente com queixas mnésicas e sintomatologia depressiva, podendo implicar, em alguns casos, uma abordagem multidisciplinar entre a Medicina Geral e Familiar, a Psiquiatria e a Neurologia.

PALAVRAS-CHAVE: Depressão; Disfunção Cognitiva; Perturbação Depressiva

1. USF Horizonte, Unidade Local de Saúde de Matosinhos, Matosinhos, Portugal. 2. USF Oceanos, Unidade Local de Saúde de Matosinhos, Matosinhos, Portugal. 3. Serviço de Neurologia, Unidade Local de Saúde de Matosinhos, Grupo de Investigação Cerebrovascular, Unidade de Investigação e Desenvolvimento Cardiovascular, Faculdade de Medicina da Universidade do Porto, Porto, Portugal. 4. Serviço de Neurologia da Unidade Local de Saúde de Matosinhos, Unidade de Investigação Epidemiológica, Instituto de Saúde Pública da Universidade do Porto, Porto, Portugal. 5. Departamento de Saúde Mental, Unidade Local de Saúde de Matosinhos, Matosinhos, Portugal. 


\section{ABSTRACT}

INTRODUCTION: About 30\% to 40\% of elderly people with depression have a reversible cognitive impairment, commonly referred to as pseudodementia, the treatable condition most often confused with dementia.

Our objective was to review the existing evidence on cognitive dysfunction in depression and the main differences in diagnosis, approach, and prognosis, in comparison with dementia.

METHODS: Literature review using the following MeSH terms: "Depressive Disorder", "Cognitive Dysfunction" and "Dementia".

RESULTS AND CONCLUSION: Many situations may present as a cognitive deficit, warranting a complete clinical history and assessment of the independent living skills of the patient. Neurological examination should always be performed, as well as the exclusion of the main organic causes of cognitive impairment. The role of the family doctor is important in the guidance of a patient with memory complaints and depressive symptoms. In some cases, this may imply a multidisciplinary approach between Family Medicine, Psychiatry and Neurology.

KEYWORDS: Cognition Disorders; Depression; Depressive Disorder

\section{INTRODUÇÃO}

A depressão major é uma doença prevalente e uma causa importante de incapacidade mundial, associada a morbilidade e mortalidade significativas. ${ }^{1}$ Ao nível dos Cuidados de Saúde Primários, 13\% a 17\% dos doentes apresentam sintomatologia depressiva, atingindo 33\% nos doentes com patologias do foro neurológico. ${ }^{2}$

Com o envelhecimento, é estimado que 10\% a 40\% das pessoas com depressão apresentem defeitos cognitivos associados. ${ }^{1}$ Por outro lado, a sintomatologia depressiva é comum na demência e ocorre em aproximadamente $30 \%$ destes pacientes. ${ }^{3}$

A depressão pode conferir um risco aumentado de demência nos 5 anos seguintes, sobretudo em pacientes idosos. ${ }^{3-6}$ Estas duas patologias partilham fatores de risco, sendo a depressão reportada como fator de risco e pródromo para a doença de Alzheimer e outras demências. $3,4,7-9^{3}$

A Organização Mundial da Saúde classificou a depressão como a doença mais onerosa do mundo em termos de anos ajustados por incapacidade total nas pessoas de meia idade..$^{10}$ Para além disso, os pacientes com depressão têm uma taxa de mortalidade mais elevada. ${ }^{11}$

O objetivo deste trabalho é rever a evidência existente sobre a disfunção cognitiva na depressão e as principais diferenças no diagnóstico, abordagem e prognóstico, comparativamente à demência.

\section{METODOLOGIA}

Foi realizada uma pesquisa bibliográfica na base de dados PubMed, com os termos MeSH "Depressive Disorder", "Cognitive Dysfunction" e "Dementia". A pesquisa foi limitada a artigos publicados em Português e Inglês. Da pesquisa inicial resultaram 291 artigos que foram, posteriormente, selecionados tendo em conta a sua pertinência, tendo sido também realizada pesquisa em cascata e consulta de livros sobre o tema. No final foram utilizados 26 artigos.

\section{RESULTADOS}

A diferenciação entre uma etiologia psiquiátrica ou neurológica para o declínio cognitivo pode ser por vezes muito difícil, devido a múltiplos fatores ${ }^{1,3,4,7,12}$ :

- As alterações cognitivas nos idosos são prevalentes e obscurecem a distinção entre o envelhecimento normal e os primeiros sinais de um processo demencial;

- A depressão pode cursar com perturbação grave da memória a curto prazo, atenção, funcionamento executivo ou velocidade de processamento, repercutindo-se nas medidas de funcionamento cognitivo global (disfunção cognitiva na depressão);

- Alguns antidepressivos associam-se a um declínio da função cognitiva, como os anticolinérgicos e as benzodiazepinas (alterações cognitivas como efeito adverso de fármacos psicotrópicos);

- A sintomatologia depressiva pode ser uma das primeiras manifestações de demência (depressão como pródromo de demência); 
- A depressão pode corresponder a uma reação ao declínio cognitivo, quando há discernimento para a perda cognitiva (depressão reativa);

- Algumas doenças neurológicas caracterizadas por declínio cognitivo progressivo, como a doença de Alzheimer e a doença de Parkinson, apresentam sintomatologia depressiva;

- A demência e a depressão, sendo muito prevalentes, podem coexistir no mesmo paciente, como duas doenças distintas ou como expressão de uma mesma doença.

Doentes com uma perturbação depressiva major podem apresentar pseudodemência., ${ }^{2,13}$ Este termo aplica-se a um leque de patologias psiquiátricas que podem mimetizar a demência orgânica, mas cuja disfunção cognitiva é potencialmente reversível com o tratamento da doença psiquiátrica de base, ao contrário do que acontece nas doenças neurodegenerativas. 5,14,15 Foram descritos os seguintes critérios de diagnóstico para pseudodemência: comprometimento intelectual em doente com doença psiquiátrica primária; características do comprometimento semelhantes às observadas nos distúrbios do sistema nervoso central; défices cognitivos reversíveis ao estado basal pré-mórbido; ausência de condição neurológica conhecida para explicar a apresentação. ${ }^{16}$ Atualmente o termo pseudodemência, quando aplicado à depressão, tem sido substituído pela designação depressão com defeito cognitivo reversível. ${ }^{13}$

A presença de disfunção cognitiva associada à depressão reduz a probabilidade de uma boa resposta ao tratamento antidepressivo. ${ }^{13}$ No entanto, quando a condição psiquiátrica se resolve ou é tratada com sucesso, estes défices geralmente revertem.,17 Os doentes com 20-30 anos raramente apresentam disfunção cognitiva na depressão. ${ }^{9}$

Apesar dos défices cognitivos na depressão serem geralmente reversíveis, existe efetivamente um risco aumentado de demência, pelo que a avaliação da cognição é importante para o prognóstico e orientação terapêutica, sobretudo quando não se verifica retorno aos níveis de funcionamento pré-mórbido. ${ }^{4,5,13,18} \bigcirc$ reconhecimento e o tratamento oportunos da depressão no idoso são importantes, não apenas para evitar que o paciente sofra as consequências da depressão progressiva ou recorrente, mas também para evitar que se realizem investigações diagnósticas desnecessárias sobre a demência. ${ }^{8,19} \mathrm{O}$ tratamento da depressão melhora a qualidade de vida do doente, independentemente de ter subjacente um defeito cognitivo, bem como pode melhorar a capacidade de colaborar na estimulação cognitiva necessária ao tratamento da demência ou da depressão. ${ }^{1}$

\section{ANAMNESE}

A história clínica é a principal fonte de informação e requer um acompanhante que conheça bem o doente para assegurar uma boa colheita da história. Devemos saber quando começaram as queixas, a sua evolução, história familiar e comorbilidades do doente. Queixas com vários anos de evolução e estáveis (não progressivas) dificilmente serão enquadráveis numa demência de etiologia degenerativa. ${ }^{19,20}$

\section{DOMÍNIOS COGNITIVOS MAIS AFETADOS}

Os sintomas cognitivos podem ser avaliados informalmente através da história clínica e objetivamente através da avaliação neurocognitiva. ${ }^{7,12,20}$

As queixas cognitivas em perturbações depressivas são geralmente consistentes, replicáveis, muitas vezes inespecíficas, apesar de clinicamente significativas e, em alguns casos, podem predizer o início de sintomas depressivos. Podem causar incapacidade psicossocial e afetam o desempenho laboral. No entanto, o padrão de incapacidade nestes doentes é moderado e não comporta a deterioração intelectual que ocorre na demência., 7,12,20

Os domínios cognitivos mais suscetíveis à disfunção na depressão são a atenção, as funções executivas e a memória (verbal e visual). 2,7,8,21

\section{ATENÇÃO}

Doentes com perturbação depressiva major referem frequentemente incapacidade de se concentrar em determinadas tarefas e de tomar decisões simples. ${ }^{2}$ A disfunção da atenção leva a uma redução da quantidade de peças de informação memorizadas, estratégias deficientes de recordação e evocação e pior desempenho na memória verbal e visual. ${ }^{12}$ Doentes demenciados e deprimidos têm uma capacidade semelhante nas tarefas que requerem atenção prolongada. ${ }^{7}$ A resposta "Não sei" e erros como confundir o sal com o açúcar estão relacionados com falta de concentração e interesse do doente deprimido e não com demência.,19

\section{FUNÇÕES EXECUTIVAS}

Doentes deprimidos cumprem melhor algumas tarefas como nomeação, repetição, leitura, sintaxe e compreensão verbal e auditiva relativamente aos doentes demenciados. No entanto, indivíduos sem depressão demonstram uma linguagem melhor que a dos doentes deprimidos, apesar de preservarema flexibilidade de raciocínio. ${ }^{7,8}$ 
Um défice residual na função mnésica e executiva parece prevalecer em alguns doentes com história de depressão e deve ser vigiado, pois uma incapacidade cognitiva irreversível após uma depressão moderada a severa, em fases avançadas da vida, é um forte preditor de demência. ${ }^{8}$

\section{MEMÓRIA}

A depressão está associada a uma maior perda de memória em situações em que é necessário maior esforço cognitivo e quando a atenção é facilmente fixada em preocupações pessoais e outros pensamentos irrelevantes para a tarefa. ${ }^{10} \mathrm{~A}$ alteração na memória de trabalho leva a uma maior dificuldade em projetar e tomar decisões. ${ }^{7}$ Os doentes deprimidos, apesar de poderem apresentar defeito cognitivo em múltiplas áreas, nomeadamente na memória episódica e na aprendizagem, têm desempenhos intactos em tarefas que requerem memória implícita, e existem outras funções de memória que também parecem ser poupadas. ${ }^{2,8,20}$

Doentes deprimidos e doentes com defeito cognitivo podem ser distinguidos com base na velocidade com que esquecem informação inicialmente codificada. Doentes com demência esquecem-se mais rapidamente comparando com doentes deprimidos e indivíduos sem depressão, que se esquecem à mesma velocidade.,20 Os doentes com demência perdem mais a memória recente do que a remota, enquanto que doentes deprimidos queixam-se da perda dos dois tipos de memória e geralmente sobrevalorizam essa perda. 2,18 Em contraste com doentes verdadeiramente com défice cognitivo, doentes deprimidos estão geralmente angustiados e têm pior prestação em alguns testes de memória do que noutros. Já os doentes com demência tendem a desvalorizar os erros e a justificá-los. Respostas que falham por pouco são típicas de défice orgânico, enquanto que "não sei" são mais típicas de depressão. ${ }^{19}$ De facto, em testes de memória, a melhoria do humor foi associada a uma meIhoria significativa da cognição. ${ }^{8}$

\section{OUTRAS DIFERENÇAS}

Os doentes deprimidos tendem a demonstrar menor variação nas suas respostas, tomando uma abordagem mais "conservadora", enquanto que doentes com demência ligeira cometem mais erros aleatórios e falsos positivos. ${ }^{8}$ A estrutura e conteúdo do discurso e orientação temporal permanecem intactas em doentes deprimidos, mas geralmente deteriora-se em doentes com distúrbios neurocognitivos. ${ }^{1}$ A presença de afasias, apraxias ou agnosias ajuda a distinguir distúrbios neurocognitivos de depressão major. ${ }^{1}$
Enquanto uma pessoa com defeito neurocognitivo pode cooperar e tentar disfarçar durante os testes, a pessoa com depressão não é, muitas vezes, cooperante, insistindo que não consegue. ${ }^{18} \mathrm{~A}$ performance em tarefas que requerem um processamento elaborado é deficiente de um modo desproporcional em indivíduos deprimidos, em comparação com a performance em tarefas automáticas. $^{7}$

Quer na depressão quer na demência, alguns sinais clínicos são a apatia, os distúrbios do sono e do apetite, a perda de interesses e o afastamento da vida social. ${ }^{7}$ A depressão pode ser distinguida da apatia na demência através de respostas emocionais expressas com pensamentos negativos acerca de si próprio, do presente e futuro, assim como desespero, desamparo e pessimismo. Doentes com depressão e "demência reversível" exibem ansiedade mais psíquica e somática, insónia terminal e perda de libido. ${ }^{13} \mathrm{Um}$ início abrupto e de curta duração não é, provavelmente, causado por demência. ${ }^{19}$ Existem várias características da história clínica que podem ajudar a diferenciar a disfunção cognitiva na depressão e na demência (Tabela 1). ${ }^{12,14,17-19}$

\section{AVALIAÇÃO NEUROCOMPORTAMENTAL E NEUROCOGNITIVA}

O principal foco da avaliação neurocomportamental é a mudança de funcionamento, que inclui capacidades cognitivas e funcionais, humor, repostas emocionais e comportamento social. ${ }^{20}$

A avaliação do estado funcional, sobre as atividades da vida diária e atividades instrumentais deve ser sempre realizada, sugerindo-se a utilização da Escala de Lawton e Brody. ${ }^{22}$

As diferenças entre indivíduos sem depressão nem défice cognitivo e doentes deprimidos podem ser suficientemente subtis para não influenciarem significativamente testes e instrumentos de avaliação cognitiva estandardizados. ${ }^{6,8}$

Contudo, a diferenciação entre depressão e os primeiros estágios da demência pode ser possível. Embora estudos anteriores tenham apontado para a incapacidade de testes neuropsicológicos para fazer essa diferenciação, dados mais recentes apoiam essa prática. ${ }^{8}$

No que diz respeito ao exame do estado mental pode ser usado o Mini Mental State Examination (MMSE), juntamente com o Teste do Desenho do Relógio, ou eventualmente o Montreal Cognitive Assessment (MoCA) em 
TABELA 1. Comparação de diferentes aspetos da história clínica entre disfunção cognitiva na depressão e a demência. ${ }^{12,14,17-19}$

\begin{tabular}{c|c|}
\hline $\begin{array}{c}\text { Disfunção cognitiva } \\
\text { na depressão }\end{array}$ & Demência \\
\hline História e curso clínico \\
\hline
\end{tabular}

Início agudo ou subagudo

Sintomas de curta duração antes da procura de ajuda médica

Progressão rápida de sintomas após instalação do quadro

História prévia comum de disfunção psiquiátrica

\section{Queixas e comportamento clínico}

Humor persistentemente triste ao longo de semanas

Sentimentos de culpa e inutilidade comuns

Pensamento suicida comum

Ansiedade e agitação piores de manhã

Início insidioso

Sintomas de longa duração antes da procura de ajuda médica

Progressão lenta de sintomas ao longo da doença

História prévia rara de disfunção psiquiátrica mas com períodos de tristeza em reação a eventos

Sentimentos de culpa e inutilidade raros

Pensamento suicida raro

Ansiedade e agitação piores ao final do dia e/ou em locais estranhos

Perda de interesse em outras atividades anteriormente prazerosas

Perda gradual de iniciativa e prazer ao longo dos anos

Poucas queixas de perda

Queixas de perda cognitiva cognitiva (embora inicialmente possa haver)

Queixas detalhadas

Queixas vagas

Enfatizam incapacidades

Escondem incapacidades

Preocupação com défices

Realçam falhanços

Minimizam e negam défices

Prazer com realizações, ainda que triviais

Pouco esforço em realizar tarefas simples

Esforço para realizar tarefas

\begin{tabular}{l|l}
\hline $\begin{array}{l}\text { Não tentam acompanhar } \\
\text { Sensação de angústia }\end{array}$ & $\begin{array}{l}\text { Dependem de notas e } \\
\text { calendários para acompanhar }\end{array}$ \\
\hline $\begin{array}{l}\text { Mudança afetiva, muitas } \\
\text { vezes difundida, profunda } \\
\text { desesperança e angústia }\end{array}$ & Afeto lábil e superficial \\
\hline $\begin{array}{l}\text { Perda de aptidões sociais cedo e } \\
\text { de forma proeminente }\end{array}$ & $\begin{array}{l}\text { Aptidões sociais } \\
\text { frequentemente mantidas, } \\
\text { mantêm "cocktail party skills" }\end{array}$ \\
\hline $\begin{array}{l}\text { Comportamento incongruente } \\
\text { com severidade da disfunção } \\
\text { cognitiva }\end{array}$ & $\begin{array}{l}\text { Comportamento compatível } \\
\text { com severidade da disfunção } \\
\text { cognitiva }\end{array}$ \\
\hline $\begin{array}{l}\text { Rara acentuação noturna da } \\
\text { disfunção }\end{array}$ & $\begin{array}{l}\text { Acentuação noturna da } \\
\text { disfunção comum }\end{array}$ \\
\hline
\end{tabular}

Características clínicas relacionadas com disfunções de memória, cognitivas ou intelectuais

Respostas típicas "Não sei”

Perda de memória recente e remota igualmente severa

Variabilidade marcada na performance de tarefas de igual dificuldade
Respostas quase certas no início

Perda de memória para eventos recentes mais severa do que para eventos remotos

Performances consistentemente pobres em tarefas de igual dificuldade

\begin{tabular}{l|l}
\multicolumn{1}{c|}{$\begin{array}{c}\text { Disfunção cognitiva } \\
\text { na depressão }\end{array}$} & \multicolumn{1}{c}{ Demência } \\
\hline $\begin{array}{l}\text { Desempenho melhor do que a } \\
\text { autoavaliação e melhora com } \\
\text { pistas }\end{array}$ & $\begin{array}{l}\text { Desempenho pior do que a } \\
\text { autoavaliação e não melhora } \\
\text { com pistas }\end{array}$ \\
$\begin{array}{ll}\text { Afasia/agnosia/apraxia } \\
\text { incomuns }\end{array}$ & $\begin{array}{l}\text { Afasia/agnosia/apraxia } \\
\text { manifestam-se coma } \\
\text { progressão da doença }\end{array}$ \\
\hline
\end{tabular}

Mudanças de apetite e de peso Perda progressiva de peso

Mais ou menos sono do que o habitual

Disrupção gradual do sono

Queixas frequentes de fadiga

Menos ativos

Lentificação psicomotora

Lentificação psicomotora variavelmente presente nas fases iniciais, mais frequente com a progressão

doentes mais diferenciados (mais de 4 anos escolaridade). É também importante que o médico utilize o teste com o qual tem mais experiência. ${ }^{19,20,23}$

O MMSE é útil para rastrear doença de Alzheimer, mas não tão útil para identificar defeito cognitivo ligeiro e outras demências. Uma versão mais extensa, o Mini-Mental State Examination Extended ( $M M X)$ requer mais tempo, mas é mais sensivel para estas patologias. ${ }^{19,21} \mathrm{O}$ MoCA é um teste de rastreio cognitivo abrangente, mas pode ser menos específico em indivíduos menos letrados. ${ }^{20,23}$ O teste de repetição das 5 palavras do MoCA é mais desafiante do que o das 3 no MMSE e inclui medidas qualitativas para recordação e reconhecimento de escolha múltipla. No entanto, requer um treino específico na sua realização e pontuação. ${ }^{20,23}$

Para a avaliação de sintomas depressivos, existem inúmeros inventários breves e listas de verificação. Os mais utilizados são a Escala de Depressão Geriátrica, a Escala de Depressão Mini-Mult, o Inventário de Sintomas Breves e o Inventário de Depressão de Beck, sendo a Escala de Depressão Geriátrica o mais utilizado e prático na consulta de Medicina Geral e Familiar. ${ }^{22}$ A brevidade e a natureza de autorrelato destes instrumentos tornam-nos fáceis de utilizar e bastante confráveis. No entanto, essas mesmas qualidades frequentemente levantam questões sobre a validade, dado que pressupõem a capacidade do paciente em responder com precisão e, muitas vezes, não avaliam uma ampla gama de sintomas afetivos e neurovegetativos, podendo subestimar a presença de depressão em idosos. ${ }^{22}$ 


\section{DIAGNÓSTICOS DIFERENCIAIS/ EXCLUSÃO DE CAUSAS ORGÂNICAS}

Diversas patologias podem cursar com perda de memória ou outros défices cognitivos, pelo que deve ser feito o diagnóstico diferencial entre várias doenças, nomeadamente do foro psiquiátrico (depressão e delírio), neurodegenerativas (doença de Alzheimer, demência vascular, demência frontotemporal e demência com corpos de Lewy/associada a doença de Parkinson), de causas metabólicas e tóxicas (hiper e hipotiroidismo, panhipopituitarismo, corticoterapia, deficiência de tiamina, anemia perniciosa, fármacos), de causas infeciosas (neurosífilis, VIH, criptococose), hidrocefalia de pressão normal, tumores intracranianos, entre outras. ${ }^{19,24-26}$

Doenças como a insuficiência cardíaca, renal e hepática, DPOC, asma, diabetes mellitus, hipertensão arterial e dor crónica devem ser rastreadas e controladas. Uma das principais etiologias de défice cognitivo é a demência vascular, pelo que em todos os doentes devem ser revistos e corrigidos todos os fatores de risco vasculares modificáveis. Deve ser sempre realizada uma revisão de toda a medicação e identificação de fármacos com potenciais efeitos laterais ao nível do sistema nervoso central e depressão como efeito adverso. ${ }^{24-26}$

O exame físico completo deve ser realizado para identificar outras eventuais patologias coexistentes que possam causar ou contribuir para um quadro demencial. Alguns achados, como a diminuição dos sons respiratórios, sons cardíacos anormais, sopros carotídeos ou alteração do volume tiroideu podem sugerir causas cardíacas, pulmonares, vasculares ou endocrinológicas de alteração do estado mental. Deve ser dada particular atenção ao exame neurológico, pesquisando défices neurológicos focais, como défices motores e sensitivos, alteração dos reflexos tendinosos, tónus muscular e alterações na marcha e equilíbrio. Os estados de privação sensorial como a hipovisão ou hipoacúsia devem ser especificamente pesquisados e abordados. ${ }^{24-26}$

No sentido de excluir as principais causas orgânicas de défice cognitivo deve ser requerido o seguinte estudo analítico: hemograma, ionograma, cálcio, função renal, hepática e tiroideia, vitamina B12 e ácido fólico, glicose, análise sumária de urina, VIH 1 e 2 e VDRL. Outras análises como a velocidade de sedimentação e eletroforese de proteínas no plasma podem ser pedidas, se suspeita de mieloma múltiplo ou causas infeciosas. 24-26

Perante doentes com mais de 65 anos, sem antecedentes de depressão prévios, sintomas depressivos despropor- cionais, persistência dos sintomas após tratamento antidepressivo ou doentes com apresentação atípica, rápida deterioração, incontinência, sinais neurológicos focais, história de traumatismo cerebral ou doenças sistémicas que afetem o cérebro (VIH, LES) deve ser pedida uma tomografia computorizada (TC) cerebral. A neuroimagem é útil no diagnóstico das demências secundárias ou atípicas, hidrocefalia, tumores, acidentes vasculares ou documentação de atrofia. Após esta investigação clínica, se for encontrada alguma causa orgânica para as queixas cognitivas do doente, esta deve ser tratada. ${ }^{24-26}$

Na ausência de causa orgânica e perante uma suspeita de etiologia depressiva, uma possível abordagem é tratar o episódio depressivo major e reavaliar as queixas mnésicas. No entanto, a espera por uma resposta para distinguir as duas entidades pode levar bastante tempo. ${ }^{24-26}$

A escolha do antidepressivo deve ser tomada caso a caso e sempre iniciada com metade da dose, pelo menos durante uma semana. A classe dos ISRS (inibidores seletivos de recaptação da serotonina) é a mais utilizada, nomeadamente a sertralina e o escitalopram. Este último é menos utilizado, devido ao teto terapêutico de 20 mg, dado os efeitos adversos cardiovasculares com doses superiores. A mirtazapina é também uma boa opção para idosos, tendo em conta o seu perfil de segurança e o aumento de apetite, muitas vezes prejudicado nesta população. Para além do tratamento farmacológico, devem ser implementadas medidas como exercício físico, estimulação cognitiva, interação social e treino cognitivo. ${ }^{17,18,24,25}$

Na ausência de melhoria de sintomas após 6 meses de terapêutica antidepressiva otimizada, o doente deve ser referenciado para consulta de Psiquiatria. Se o doente mantiver défices ou queixas cognitivas após 6 meses de tratamento eficaz da depressão, ou se tiver história sugestiva de demência, deve ser referenciado para Neurologia. ${ }^{17,18,24,25}$

\section{CONCLUSÃO}

A depressão é a causa mais comum de disfunção cognitiva em idosos e é também a condição tratável mais comummente diagnosticada erroneamente como demência. Estudos epidemiológicos demonstraram que doentes com história de depressão têm um risco duas vezes superior de desenvolver demência irreversível, e uma parte dos doentes com depressão e demência reversível irão progredir para demência irreversível em 2 a 3 anos. Portanto, tendo em conta este risco aumentado de demência irreversível, torna-se vital o reconheci- 
mento e o tratamento oportunos da depressão e avaliação rigorosa da cognição nos doentes deprimidos.

Num doente que se apresenta com queixas mnésicas, a história clínica é a principal fonte de informação, sendo importante saber quando começaram as queixas mnésicas, a sua evolução, fatores hereditários e comorbilidades. É também importante avaliar o estado funcional, as mudanças comportamentais e indagar a presença de sintomas depressivos. $\bigcirc$ exame neurológico sumário deve ser sempre realizado, assim como excluídas as principais causas orgânicas de défice cognitivo.

A orientação do doente com queixas mnésicas e sintomatologia depressiva vai depender das alterações presentes no conjunto "história clínica + exame físico + exames complementares de diagnóstico" e, em alguns casos, vai implicar uma abordagem multidisciplinar entre a Medicina Geral e Familiar, a Psiquiatria e a Neurologia.

\section{APRESENTAÇÕES}

A presente revisão foi apresentada, sob a forma de comunicação oral, no $37^{\circ}$ Encontro Nacional de MGF da Associação Portuguesa de Medicina Geral e Familiar.

\section{RESPONSABILIDADES ÉTICAS}

CONFLITOS DE INTERESSE: Os autores declaram não possuir conflitos de interesse.

SUPORTE FINANCEIRO: O presente trabalho não foi suportado por nenhum subsídio ou bolsa.

PROVENIÊNCIA E REVISÃO POR PARES: Não comissionado; revisão externa por pares.

\section{ETHICAL DISCLOSURES}

CONFLICTS OF INTEREST: The authors have no conflicts of interest to declare.

FINANCIAL SUPPORT: This work has not received any contribution grant or scholarship.

PROVENANCE AND PEER REVIEW: Not commissioned; externally peer reviewed.

\section{REFERÊNCIAS}

1. Schulz P, Arora G. Depression. Continuum: Lifelong learning. Neuroloy. 2015;21:756-71. doi: 10.1212/01.CON.0000466 664.35650.b4.

2. Rakofsky J, Rapaport M. Mood Disorders. Continuum. 2018;24:804-27. doi: 10.1212/CON.0000000000000604.

3. Barnes D, Alexopoulos G, Lopez O, Williamson J, Yaffe K. Depressive symptoms, vascular disease, and mild cognitive im- pairment. Arch Gen Psychiatry. 2006;63:273. doi: 10.1001/ archpsyc.63.3.273.

4. Bennett S, Thomas A. Depression, and dementia: Cause, consequence or coincidence? Maturitas. 2014;79:184-190. doi: 10.1016/j.maturitas.2014.05.009.

5. Keren R. Will the Real Pseudodementia Please Stand Up?. The Canadian Review of Alzheimer's Disease and Other Dementias. 2008;11:11-14.

6. Onyike CU. Psychiatric Aspects of Dementia. Continuum. 2016;22:600-14. doi: 10.1212/CON.0000000000000302.

7. Leposavic I, Leposavic L, Gavrilovic P. Depression vs. Dementia: A comparative analysis of neuropsychological functions. Psihologija. 2010;43:137-153.

8. Kang H, Zhao F, You L, Giorgetta C, Sarkhel S, Prakash R. Pseudo-dementia: A neuropsychological review. Ann Indian Acad Neurol. 2014;17:147-54. doi: 10.4103/0972-2327.132613. PMCID: PMC4090838.

9. Kobayashi T, Kato S. Depression-dementia medius: Between depression and the manifestation of dementia symptoms. Psychogeriatrics. 2011;11:177-182. doi: 10.1111/j. 1479-8301.2011.00363.x.

10. Gotlib I, Joormann J. Cognition and Depression: Current Status and Future Directions. Annu Rev Clin Psychol. 2010;6:285312. doi:10.1146/annurev.clinpsy.121208.131305.

11. Rosano C, Ikram MA, Ganguli M. Preface. Handb Clin Neurol. 2016;138:ix-x. doi: 10.1016/B978-0-12-802973-2.09987-0.

12. McClintock SM, Husain MM, Greer TL, Cullum CM. Association between depression severity and neurocognitive function in major depressive disorder: A review and synthesis. Neuropsychology. 2010;24:9-34. doi: 10.1037/a0017336.

13. Morimoto SS, Kanellopoulos D, Manning KJ, Alexopoulos GS. Diagnosis and treatment of depression and cognitive impairment in late life. Ann N Y Acad Sci. 2015;1345:36-46. doi: 10.1111/nyas.12669.

14. Fisman M. Pseudodementia. Prog Neuropsychopharmacol Biol Psychiatry. 1985;9:481-4. doi: 10.1016/02785846(85)90005-3.

15. Connors M, Quinto L, Brodaty H. Longitudinal outcomes of patients with pseudodementia: a systematic review. Psychol Med. 2018 15;1-11. doi: 10.1017/S0033291718002829.

16. McIntyre R, Cha D, Soczynska J, Woldeyohannes H, Gallaugher L, Kudlow P, et al. Cognitive deficits and functional outcomes in major depressive disorder: determinants, substrates, and treatment interventions. Depress Anxiety. 2013;30:515-27. doi: 10.1002/da.22063.

17. Alzheimer's \& Dementia Weekly. Pseudodementia is Often Curable [homepage on the internet]; 2016 [accessed 1 Oct 2020]. Available from: http://www.alzheimersweekly. com/2016/05/pseudodementia-is-often-curable.html.

18. Kramer B. Depressive pseudodementia. Compr Psychiatry. 1982;23:538-544. doi: 10.1016/0010-440x(82)90045-1.

19. Kaufer D. Neurobehavioral Assessment. Continuum. 2015; 21:597-612. doi: 10.1212/01.CON.0000466655.51790.2f.

20. Airaksinen E, Larsson M, Lundberg I, Forsell Y. Cognitive functions in depressive disorders: evidence from a population-based study. Psychol Med. 2004;34:83-91. doi: 10.1017/ s0033291703008559.

21. Sahin S, Önal TU, Cinar N, Bozdemir M, Cubuk R, Karsidag S. Distinguishing Depressive Pseudodementia from Alzheimer Disease: A Comparative Study of Hippocampal Volumetry and Cognitive Tests. Dement Geriatr Cogn Dis Extra. 2017;7:2309. doi: 10.1159/000477759. 
22. Núcleo de Estudos de Geriatria da Sociedade Portuguesa de Medicina Interna. Avaliação Geriátrica. [homepage on the internet]. Available from: https://www.spmi.pt/wp-content/ uploads/GERMI_36.pdf.

23. Freitas S, Simões M, Santana I. Montreal Cognitive Assessment (MoCA): Pontos de corte no Défice Cognitivo Ligeiro, Doença de Alzheimer, Demência Frontotemporal e Demência Vascular. Sinapse. 2014;14:18-30.

24. Saraiva C, Cerejeira J. Psiquiatria fundamental. Coimbra: LIDEL; 2014.

25. Direção Geral da Saúde. Norma da Direção-Geral da Saúde nº 053/2011 atualizada a 27/12/2011. Abordagem Terapêutica das Alterações Cognitivas. Lisboa: DGS; 2011.

26. Ribeira S, Ramos C. Avaliação inicial da demência. Rev Port Clin Geral. 2004;20:569-77. 